\title{
Dynamical Simulations of Transmission Kikuchi Diffraction (TKD) Patterns
}

\author{
Elena Pascal ${ }^{1}$, Saransh Singh ${ }^{2}$, Ben Hourahine ${ }^{1}$, Carol Trager-Cowan ${ }^{1}$ and Marc De Graef ${ }^{2}$ \\ ${ }^{1}$ Dept. of Physics, SUPA, University of Strathclyde, Glasgow, G4 0NG, UK. \\ 2 Dept. of Materials Science and Engineering, Carnegie Mellon Univ., Pittsburgh PA 15213, USA.
}

Truly nanostructured materials pose a significant spatial resolution challenge to the conventional Electron Backscatter Diffraction (EBSD) characterization technique. Nevertheless, the interaction volume can be reduced by the use of electron transparent samples and the acquisition of electron backscatterlike patterns (EBSP) in transmission mode instead. These transmission Kikuchi diffraction (TKD) patterns are typically acquired by mounting a thin foil, similar to transmission electron microscopy (TEM), and tilting it at a slight angle $\left(20^{\circ}-30^{\circ}\right)$ from horizontal towards a standard EBSD camera.

While TKD has recently gained attention as a high resolution orientation mapping technique [1], indexing of TKD patterns has mostly been performed using the standard EBSD approaches, accounting for the difference in geometry. Only limited work has been done in terms of dynamical TKD pattern simulations. Since it is known from experiments that the sample thickness directly influences the sharpness of the patterns [2] as well as the pattern details, we can expect a transmission model for TKD patterns to show quantitative differences compared to EBSD pattern predictions. We propose the following formulation for the probability that an electron of energy $E$ will leave the foil in the direction $\hat{\mathbf{k}}$ after Rutherford scattering from an individual atom located at a distance $z$ from the exit surface:

$$
P(\hat{\mathbf{k}})=\sigma \int_{E_{\min }}^{E_{\max }} \mathrm{d} E \int_{0}^{z_{0}(E)} \mathrm{d} z \bar{\lambda}_{\hat{\mathbf{k}}}(E, z)\left|\Psi_{\hat{\mathbf{k}}}\left(\mathbf{r}_{j} ; E, z\right)\right|^{2} .
$$

Here, $\sigma$ is the Rutherford scattering cross section; $E_{\min / \max }$ are the minimum and maximum energies considered in the calculation; $z$ is the distance between the scattering site and the sample surface, measured along the exit direction; $z_{0}(E)$ is the maximum distance to be considered; $\bar{\lambda}_{\hat{\mathbf{k}}}(E, z)$ is a weighting function describing the fraction of incident electrons (per unit energy and per unit length) of energy $E$, originating a distance $z$ from the sample surface and traveling in the direction $\hat{\mathbf{k}}$.

In contrast to the EBSP signal, which provides information about a volume close to the illuminated top surface of the sample, the majority of the electrons contributing to a TKD pattern 'originate' from the bottom region of the sample; they are inelastically scattered inside the sample before reaching the bottom portion of the foil and escaping via a final elastic (Rutherford) scattering event. The formation of these forward scattered escaping electrons is a stochastic process encoded by the function $\bar{\lambda}_{\hat{k}}$, and determined with a Monte Carlo (MC) trajectory simulation. Fig. 1 shows stereographic projections of the exit electron distribution for a $100 \mathrm{~nm} \mathrm{Ni}$ foil tilted at $30^{\circ}$ as a function of the exit energy. The exit energy range depends strongly on sample thickness and atomic number, as illustrated in Fig. 2 for $\mathrm{Ni}$ and $\mathrm{Si}$; the mean exit energy decreases with increasing sample thickness. This, in turn, must give rise to both broadening and blurring of the Kikuchi bands compared to those observed in an EBSD pattern. This is illustrated in Fig. 3, which shows line profiles across a Kikuchi band, shown on the left, for different sample thicknesses. We will demonstrate dynamically simulated TKD patterns for a number of material systems and compare them with experimental observations; equation 1 provides a good forward model for TKD pattern simulations.

\section{References}

[1] P W Trimby, et al, Acta Materialia 62 (2014) p. 69. 
[2] Y Z Wang, et al, Journal of Microscopy, 264, p. 34.

[3] P G Callahan and M De Graef, Microscopy and Microanalysis, 19, p. 1255.

[4] Research supported by ONR \# N00014-16-1-2821. The authors acknowledge use of the Materials Characterization Facility at Carnegie Mellon University supported by grant MCF-677785.

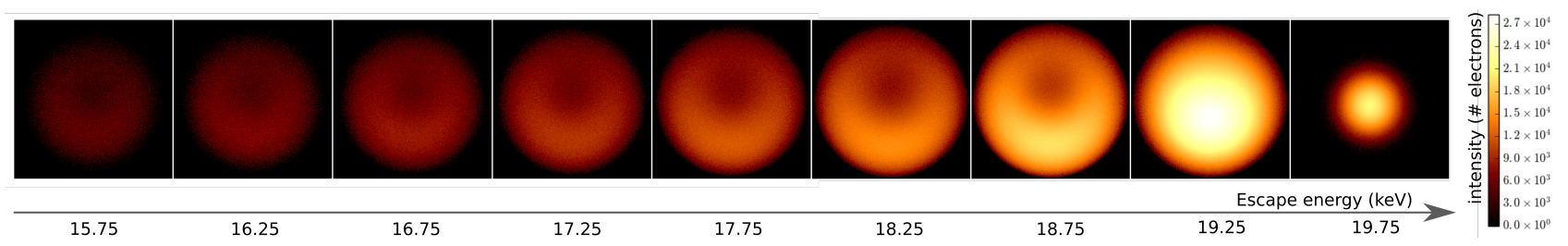

Figure 1. TKD electron distribution for different escape energies for a $100 \mathrm{~nm} \mathrm{Ni}$ foil tilted at $30^{\circ}$ from horizontal. 30000 electrons were simulated with incident energy of $20 \mathrm{keV}$.
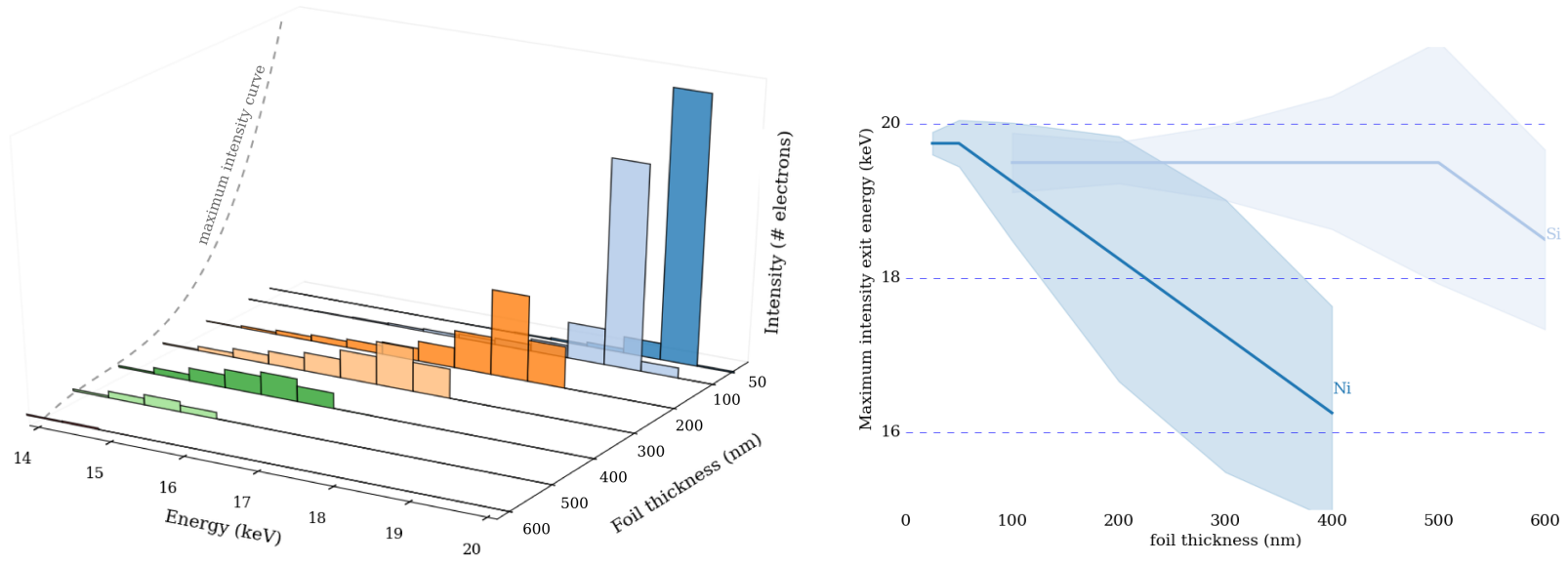

Figure 2. The shift of the electron energy distribution peak with the film thickness for Ni is shown on left. On the right the broadening of the energy distribution peak with sample thickness is depicted as a standard deviation envelope for both $\mathrm{Si}$ and $\mathrm{Ni}$.
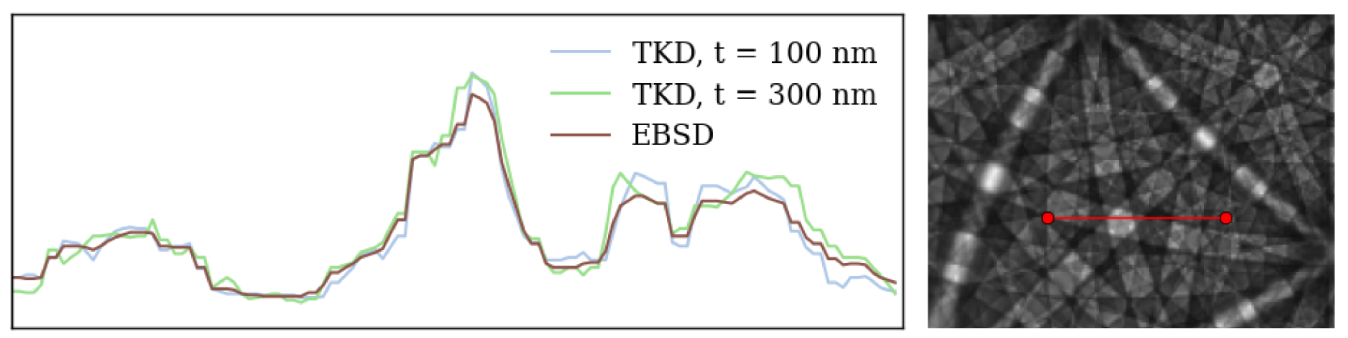

Figure 3. Intensity line scan (red line on the right) comparison between EBSD (dark red line) and TKD patterns (blue line for a $100 \mathrm{~nm}$ thick sample and green line for a $300 \mathrm{~nm}$ sample) simulated for Ni in same sample geometry. The TKD pattern not only show higher contrast features, but also finer details than the EBSD one. 